\section{Prepare for Post-COVID-Pandemic Polio Problems}

The current crisis of the COVID-19 pandemic will, most likely, abate in due course as herd immunity builds up in all affected countries [1]. Among all public health projects that are stalled now, global polio eradication is a critical one and it ought to be put back on rail. However, we foresee formidable obstacles and propose remedial actions.

At a global level, on 24 March, 2020, the leadership of global polio eradication recommended suspending all OPV campaigns until at least second half of the year to prevent spread of SARS-CoV-2 [2]. Globally, vaccine derived polio virus (VDPV) type 2 outbreaks were not getting contained by OPV campaigns [3], and they may expand and spread widely as lockdowns are lifted in the affected countries. We may see resurgence of wild-virus polio in Afghanistan and Pakistan.

In India, all activities under the Universal immunisation programme (UIP) were stalled at the start of the lockdown, and later resumed in different parts of the country amidst patchy availability of public transport. There are consequences, affecting all vaccinepreventable diseases, but here we focus on the problems of polio as it is under eradication mode. The major risk in India and in all countries still using bivalent OPV with types 1 and 3, is the likelihood of emergence of VDPVs type 1 and type 3 [4], on account of long periods of no immunization following its widespread use until JanuaryFebruary 2020, including national pulse immunization in January, 2020. It is to prevent the emergence of VDPVs that India's UIP had been keeping up high herd immunity in children through OPV under UIP plus annual pulse campaigns twice annually, in spite of eradication of wild polioviruses in 2014. As polio immunization is suspended,
India must anticipate the emergence and circulation of VDPVs. We will know the full picture only after the lockdown is lifted and surveillance for acute flaccid paralysis (AFP) and for polioviruses is resumed.

We feel that the safest strategy to mitigate this impending risk of VDPVs is to prepare for intensified immunization with IPV, aiming to not reintroduce OPV in children in India after achieving high IPV coverage. Full IPV immunization requires priming and boosting. If a high proportion of children are protected with full immunizing schedule of IPV adding booster dose(s), the chances of VDPV emergence will be minimized and any emerged VDPV transmission can be intercepted.

It is time for us to make these policy decisions after dialogue with stake-holders, supported by science and evidence accumulated in the country over the past 6 decades - and choose the path which is in the best interests of our children.

Funding: None; Competing interests: None stated. Published online: May 09, 2020; PII: S097475591600178

\section{T JACOB JOHN ${ }^{1}$ AND DHANYA DHARMAPALAN ${ }^{2 *}$ \\ ${ }^{1}$ Christian Medical College, Vellore, Tamil Nadu; and ${ }^{2}$ Apollo Hospitals, CBD Belapur, Navi Mumbai, India. *drdhanyaroshan@gmail.com}

\section{REFERENCES}

1. John TJ. Will Coronavirus pandemic eventually evolve as Pan-endemic? Curr Sci. 2020;118:855-6.

2. Global Polio Eradication Initiative. Call to action to support COVID-19 response. Available from: http:// polioeradication.org/news-post/call-to-action-to-supportcovid-19-response. Accessed April 25, 2020.

3. Centers for Disease Control and Prevention. Update on Vaccine-Derived Poliovirus Outbreaks - Worldwide, July 2019-February 2020; MMWR. 2020;69:489-95.

4. World Health Organization. Polio outbreak in Papua New Guinea. Geneva: WHO; 2018 [cited 2019 Oct 7]. Available from: https://www.who.int/westernpacific/emergencies/ papua-new-guinea-poliovirus-outbreak. Accessed May 8, 2020. 